\title{
Extended Amygdala and Emotional Salience: A PET Activation Study of Positive and Negative Affect
}

\author{
Israel Liberzon*, ,2, K Luan Phan', Laura R Decker and Stephan F Taylor' \\ 'Department of Psychiatry, University of Michigan, Ann Arbor, MI, USA; ${ }^{2}$ Psychiatry Senvice, Veterans Affairs Medical Center, Ann Arbor, MI, USA
}

Functional neuroimaging studies have implicated amygdaloid and basal forebrain regions, including sublenticular extended amygdala (SLEA), in the mediation of aversive emotional responses. However, it is not clear whether SLEA responds to 'aversiveness' or to general stimulus salience. We predicted that both pleasant and aversive stimuli would activate this region. Using $\left[{ }^{15} \mathrm{O}\right]$ water PET, we studied 10 healthy subjects while viewing pleasant, aversive, neutral, and blank images. Each subject underwent eight scans, which were processed and averaged with standard statistical methods. Both positive and negative stimuli activated regions in SLEA. Both positive and negative content activated the visual cortex, relative to neutral content. Aversive stimuli deactivated the left frontal pole, relative to positive and neutral stimuli. These findings demonstrate that both positive and negative emotional content evokes processing in the sublenticular/ extended amygdala region, suggesting that this region is involved in general emotional processing, such as detection or attribution of salience.

Neuropsychopharmacology (2003) 28, 726-733. doi:I0.I038/sj.npp. I 300 II3

Keywords: emotion; SLEA; cortex; limbic; neuroimaging

\section{INTRODUCTION}

Several groups, including our own, have reported activation in sublenticular extended amygdala (SLEA) in response to aversive (AV) stimuli, consistent with animal and human lesion studies. Until recently, imaging studies have often associated discrete forebrain regions (such as amygdala and nucleus accumbens) with emotional responses (Breiter $e t a l$, 1996; Liberzon et al, 2000; Morris et al, 1997; Phillips et al, 1997; Whalen et al, 1998); potentially obscuring an important anatomical continuity. Studies of basic neuroanatomy have demonstrated similar architectonics, connectivity, and neurotransmitter populations in the amygdaloid nuclei, sublenticular nuclei, and the nucleus accumbens, meriting the unifying designation of 'SLEA' (Alheid and Heimer, 1988; de Olmos and Heimer, 1999; Heimer et al, 1997a). This recent reconceptualization of basal forebrain anatomy is particularly relevant to the study of emotions. For instance, the core of the nucleus accumbens, at the most rostral extension of SLEA, plays a key role in the processing of behavioral rewards (for recent reviews see Kalivas and Nakamura, 1999; Koob, 2000), and the continuity between the nucleus accumbens and the amygdaloid nuclei suggests

\footnotetext{
This work was previously presented at the Human Brain Mapping Society annual meeting, San Antonio, Texas, June 2000.

* Correspondence: Dr I Liberzon, UH-9D Box 0I I8, I500 E. Medical Center dr, Ann Arbor, Ml 48I09-0 I I8, USA, Tel: + 7347636497 , Fax: +I 734936 7868, E-mail: liberzon@umich.edu

Received 12 February 2002; revised 30 October 2002; accepted I November 2002

Online publication: 15 November 2002 at http: //www.acnp.org/ citations/Npp I | I502429
}

the involvement of various elements of SLEA in the processing of both appetitive and aversive stimuli.

The participation of these regions in both aversive and appetitive responses might seem at odds with the view that distinct regions comprising SLEA respond to a particular stimulus valence, for example, the amygdala for aversive responses such as fear (Paradiso et al, 1999) and the nucleus accumbens for appetitive responses such as reward (Elliott et al, 2000). However, an extensive animal literature provides ample evidence that the amygdaloid nuclei also respond to appetitive, as well as aversive, stimulus qualities (Davis, 1992; Gallagher and Schoenbaum, 1999; Holland and Gallagher, 1999; Kling and Brothers, 1992; Rasia-Filho et al, 2000 ), and recent findings implicate the shell of the nucleus accumbens in the processing of fear (Reinolds and Berridge, in press). In addition, in vivo neuroimaging studies have reported amygdaloid activation in response to positive (POS) and appetitive stimuli (Lane et al, 1997b; Zalla et al, 2000). Whalen et al (1998) demonstrated that happy faces activated substantia inominata, SLEA structure functionally contiguous with the amygdala, and Hamann et al (Hamann and Mao, 2002) reported amygdaloid activation in response to positive verbal stimuli.

Thus, it is reasonable to propose that structures within SLEA, rather than activating to the specific aversive characteristics of stimuli, respond to more general qualities, such as stimulus salience. A similar interpretation has been proposed by Whalen et al (1998) and by Davis and Whalen (2001) for activation of the substantia inominata in response to emotional faces. Here, we define salient stimuli as those that engage processing because of potential importance for basic biological drives and psychological 
needs. In prior studies, we have demonstrated activation in SLEA with both aversive and nonaversive (NA) stimuli and hypothesized that the entire region processes emotional salience, irrespective of stimulus valence (Liberzon et al, 2000; Taylor et al, 1998). Unlike emotional facial expressions used by Whalen et al (1998), which engage the recognition of emotions and/or the detection of dangerous/ ambiguous cues, the visual stimuli we have used, from the International Affective Picture System, are intended to directly elicit the emotion (perception of the emotional stimuli and the experience of emotion). It is not clear whether or not these evocative stimuli will also activate SLEA, irrespective of valence. To further explore the role of the extended amygdala in emotional responses, we examined regional cerebral blood flow ( $\mathrm{rCBF}$ ) responses to pleasant and aversive visual stimuli. Based on the evidence described above, we predicted that positive salient stimuli, as well as aversive stimuli, would activate SLEA.

In addition to basal forebrain activation, emotionally salient stimuli produce consistent modulation of the visual cortex (Kosslyn et al, 1996; Lane et al, 1997b; Lang et al, 1998; Liberzon et al, 2000; Taylor et al, 2000) and the dorsal medial prefrontal cortex (Gusnard et al, 2001; Lane et al, 1997a; Teasdale et al, 1999). These cortical regions are highly interconnected with the SLEA (Ghashghaei and Barbas, 2001) and potentially constitute a functional network that is activated in response to emotional stimuli. Similar to the question raised about SLEA, we ask: Are these regions responding to 'aversiveness' (the predominate choice for emotionally evocative stimuli) or to the salience of the stimuli? Focusing on the visual cortex, Lang et al (1998) addressed this issue in a study with aversive and pleasant visual images. They found visual cortical modulation to both positive and negative stimuli, relative to nonaversive stimuli. However, for the rMPFC, we are not aware of studies using both types of stimuli. In a previous study we have noted dMPFC activation to salient stimuli, in association with cognitive processing of the stimuli. The inverse relations observed between the activations in SLEA and in the dMPFC suggested a possible role for dMPFC in cognitive modulation of emotional responses (Taylor et al, in press). Therefore, we predicted that both positive and aversive emotional content would modulate activity in visual cortex and dMPFC.

\section{METHODS}

\section{Subjects}

A total of 10 healthy volunteers (six men, four women, age $27.5 \pm 8.6$ years) without history of major medical, neurological, or psychiatric illness (negative SCID, Spitzer et al, 1988) were recruited through advertisements. All subjects gave written informed consent after explanation of the experimental protocol, as approved by the local Institutional Review Board. Subjects were compensated 150 fortheirparticipation.

\section{Stimuli}

Three sets of 18 images each were selected from the standardized International Affective Picture System (IAPS), supplemented by our collection (approximately 10\% of all images). The three sets consisted of POS pictures (animals, children, food, humor), AV pictures (facial mutilation, wounds, dead bodies), and NA pictures (faces with neutral expressions, benign scenes). A fourth condition (blank condition, BL) consisted of a white fixation cross on a gray background (five different shades). Since processing of faces recruits specific brain regions (Dolan et al, 1996; Haxby et al, 1996), we matched the sets (POS, AV, NA) with respect to faces and human figures. In addition, to avoid possible differential effects of color composition (ie more red in aversive images), all images were transformed into black and white, and luminance was balanced across the four sets with Photoshop 4.0 (Adobe Systems).

\section{Psychophysiology}

Skin conductance and horizontal electro-oculogram (EOG) were recorded using an MP-100 psychophysiological monitoring system (BioPac Systems). Skin conductance was recorded using $\mathrm{Ag} / \mathrm{AgCl}$ electrodes filled with isotonic electrolyte jelly (KY jelly) attached to the volar surface of the second phalanx of the first and third fingers of the left hand. Waveform peak and integral during image presentation were analyzed, relative to baseline skin conductance (immediately prior to image presentation).

Horizontal EOG was recorded by means of $\mathrm{Ag} / \mathrm{AgCl}$ electrodes affixed to the lateral canthus. Waveforms were corrected for drift, mean subtracted, and divided by the magnitude of a calibration signal to normalize values. The percentage of time gaze fell outside the bounds of the monitor screen was calculated. The amount of eye movement was quantified as the standard deviation of EOG.

\section{Experimental Protocol}

During the scanning sessions, subjects viewed two blocks of images with either pleasant, aversive, or neutral content. Images were displayed consecutively for $5 \mathrm{~s}$ each with no interstimulus interval, on a computer monitor suspended $40 \mathrm{~cm}$ from the subject at a $15^{\circ}$ viewing angle, using SuperLab (Cedrus, Inc.). The first block consisted of neutral stimuli without image acquisition, allowing subjects to adjust to the scanner. During this block, the injection of a small dose $(8 \mathrm{mCi})$ of radiotracer determined arrival time in the brain. The timing block was followed by two identically ordered sets of four blocks from each of the four conditions (POS, AV, NA, BL), with order counterbalanced in a partial Latin square. Subjects were instructed to focus on feelings that they experienced while viewing the pictures. During stimulus presentation, subjects rated images according to a 5 -point scale in which 5 corresponded to very unpleasant, and 1 corresponded to very pleasant. For the blank condition, subjects said ' 3 ' every time the gray screen changed, which occurred every $5 \mathrm{~s}$.

\section{PET Data Acquisition}

The subjects underwent PET scans in a Siemens CTI 931-08/ 12 scanner (CTI Inc., Knoxville, TN), which acquires 15 slices over an axial length of approximately $10 \mathrm{~cm}$. A transmission scan was acquired by external ${ }^{68} \mathrm{Ge} /{ }^{68} \mathrm{Ga}$ ring 
sources. Eight emission scans, separated by $10 \mathrm{~min}$, were acquired for each subject. Subjects were given an i.v. bolus injection of $50 \mathrm{mCi}$ of $\left[{ }^{15} \mathrm{O}^{\mathrm{H}} \mathrm{H}_{2} \mathrm{O}\right.$. Data were collected in a single $60 \mathrm{~s}$ frame beginning $5 \mathrm{~s}$ after the arrival of the radioactivity in the brain. Stimuli presentation began $5 \mathrm{~s}$ prior to image acquisition, and continued for the first $30 \mathrm{~s}$ of each scan (uptake phase of the tracer), so as to maximize changes in the PET signals associated with the task of interest (Cherry et al, 1993). The second $30 \mathrm{~s}$ of each scan contained blank stimuli from the blank condition.

\section{PET Data Analysis}

Analysis of the PET data first employed a standardization process that enabled image averaging within and across subjects. Automated routines proportionally normalized images to the mean of global activity (arbitrarily set to 1000), and individual images within each subject were coregistered to the fourth scan to correct for head movement between scans (usually less than $2 \mathrm{~mm}$ translation and less than $2^{\circ}$ rotation). A nonlinear transformation was applied to each image to standardize individual anatomy (Minoshima et al, 1992, 1993, 1994). Standardized images were smoothed with a 3D Gaussian filter, yielding a final, effective FWHM of around $14 \mathrm{~mm}$. Difference images were made by subtracting scans obtained in different conditions, and were expressed as $Z$-scores, using the between-subjects variance (Friston et al, 1991; Worsley et al, 1992).

We searched for significant activations on the basis of regional a priori hypotheses as well as an image-wide search unconstrained by regional predictions. To test our specific $a$ priori hypothesis, we searched for activation foci, thresholded at $p<0.01$ uncorrected $(Z>2.33)$, in the anatomically constrained region covering SLEA bilaterally. We defined the region bounded by the following coordinates from the Talairach and Tournoux atlas: anterior/posterior +15 to $-10 \mathrm{~mm}$; inferior/superior -20 to $-4 \mathrm{~mm}$; right/left +25 to $-25 \mathrm{~mm}$. For the visual cortex and dMPFC, larger regions of the brain than SLEA, we adopted a more conservative of threshold $p<0.001$ for reporting activation foci. Outside a priori regions, we reported activation that exceeded a corrected probability of $p<0.05$, corresponding to a $Z$-score of around 4.4 for our image sets (28). For post hoc analysis, we used $13.5 \mathrm{~mm}$ in diameter, spherical volumes of interest (VOI) to sample individual images at the coordinate locations of selected peaks. The volume of the VOI does not allow one, however, to conclude with certainty that activation peaks within VOI represent exactly the same region, but could originate from two adjacent locations within the VOI. For two subjects, head position precluded analysis of foci in the amygdala and SLEA; therefore, these subjects were not included in the analysis of regions where brain activity was not sampled.

\section{RESULTS}

\section{Behavioral and Psychophysiologic Results}

Averaged stimulus ratings collected during the PET scans confirmed that our subjects subjectively experienced negative, neutral, and positive affects $(4.69 \pm 0.25$, $2.67 \pm 0.46,1.01 \pm 0.05$, respectively) as intended (Figure 1).
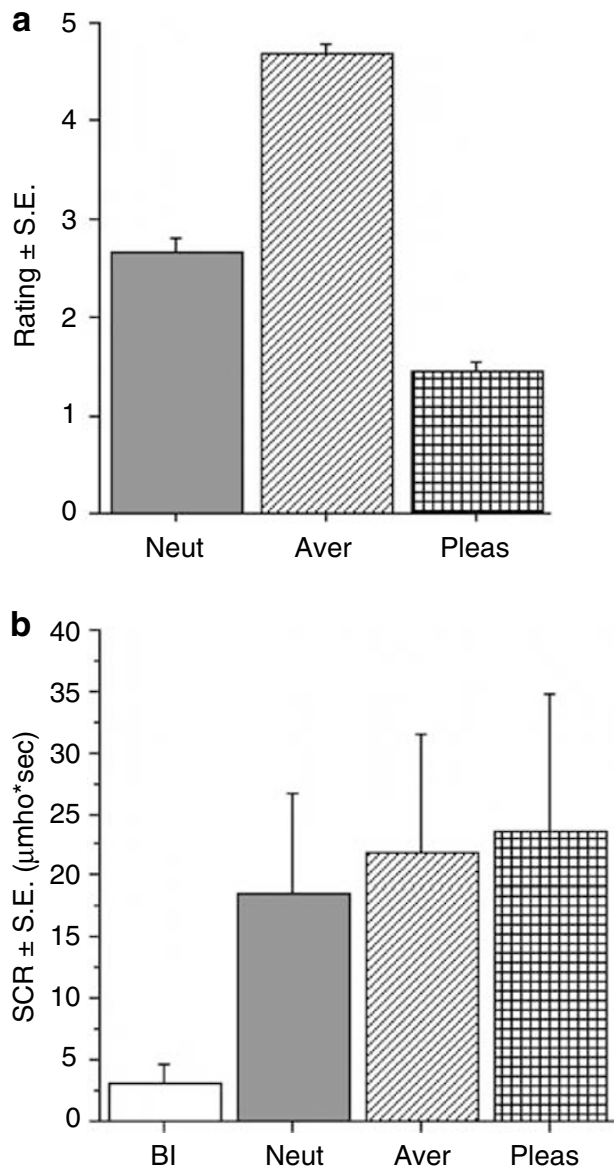

Figure I Ratings of subjective experience and skin conductance responses. While viewing stimuli, subjects rated pictures on a 5-point scale, with 3 being 'neutral'. NA stimuli were rated as overall slightly more positive than 'neutral'. The blank condition was not rated. Skin conductance responses are presented as an integral of the waveform during picture presentation, relative to a I s baseline prior to viewing pictures.

Analysis of EOG data confirmed that subjects scanned the pictures and did not divert their gaze from the aversive pictures. There were more lateral eye movements (trend level) during aversive stimuli presentation $(0.152 \pm 0.013$ in units of SD) as compared to nonaversive $(0.132 \pm 0.039)$ and positive stimuli $(0.105 \pm 0.006)$ (repeated measure ANOVA effect of condition $\left.\mathrm{F}_{(2,7)}=10.34, p=0.08\right)$. Neutral, negatively, and positively valenced stimuli elicited significant increases in skin conductance $(21.9 \pm 9.6$ and $23.5 \pm 11.2$, integrated response in $\mu$ mho \pm SD), respectively, as compared to the baseline, which did not differ however between these conditions (repeated measure $\left.\operatorname{ANOVA~}_{(2,7)}=0.89, p=0.45\right)$.

\section{rCBF Results}

Activation in limbic regions. As predicted, emotional stimuli, both positive and aversive, activated the SLEA region compared to nonaversive stimuli (see Figure 2; AV-NA: $x, y, z=8,10,-7, Z=3.28$; POS-NA: $-1,5,-4$, $Z=2.81$ ); while the peaks of the activation foci differed slightly, our hypothesis stated that we would activate the same region for both the POS-NA and the AV-NA 


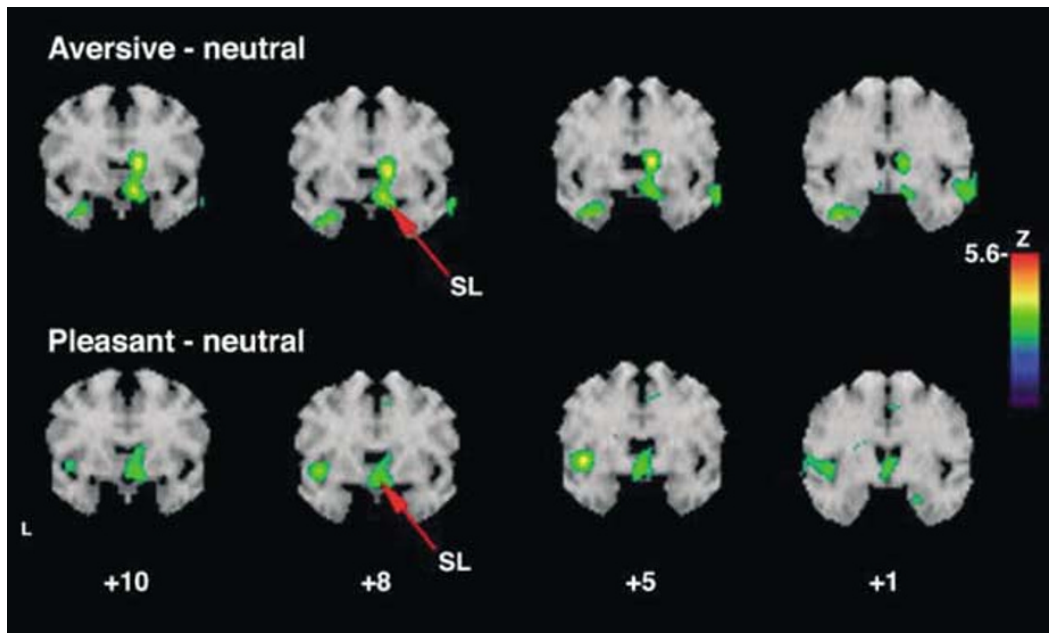

Figure 2 Activity increases in limbic regions while viewing aversive and positive stimuli compared to nonaversive stimuli. The figure shows the activity increase for aversive pictures (upper row) and positive pictures (lower row) relative to the nonaversive condition in coronal planes. For both comparisons, activation is depicted as $Z$-scores on the color scale, displayed for $Z>1.65$ (uncorrected probability of $p<0.05$ ), mapped onto a reference MRI in atlas coordinates. Numbers below the bottom row correspond to $\mathrm{mm}$ anterior/posterior of the anterior commissure. SL: the red arrow points to the peak activity in the SLEA region.

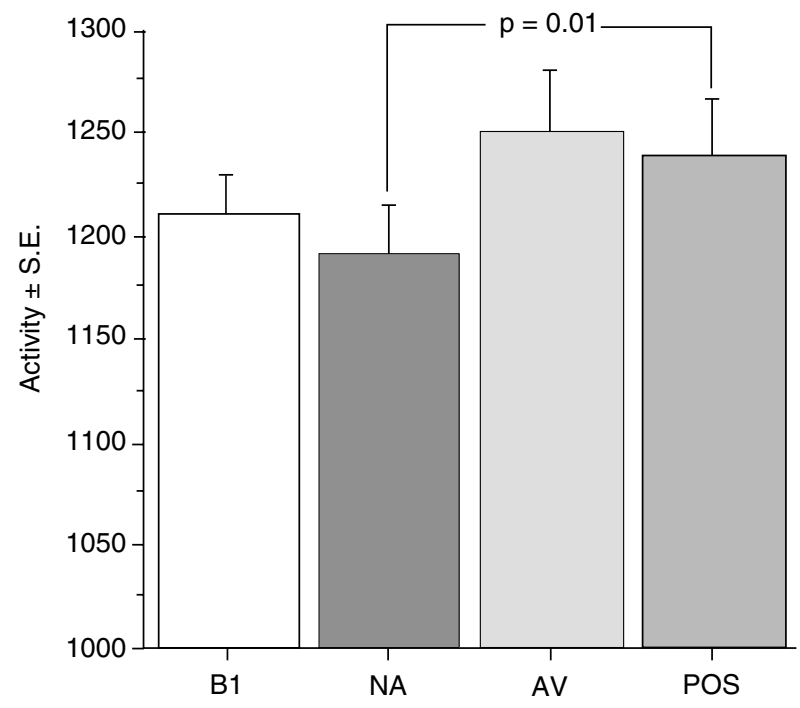

Figure 3 Extended amygdala activity in aversive, nonaversive, and positive conditions. The figure depicts activity in $13.5 \mathrm{~mm} \mathrm{VOI}$ centered on the coordinates of activation peak found in $A V-N A$ subtraction. Significantly greater activity $(p=0.0 \mathrm{I})$ was present during the positive as compared to nonaversive condition. $Y$-axis represents counts normalized to the mean of global activity, arbitrarily set to 1000

comparisons. Therefore, we used the AV-NA peak to define an independent ROI in order to test the POS-NA comparison for activation. Post hoc volume of interest analysis confirmed significant activation in the POS-NA comparison $(t=3.44, \mathrm{df}=7, p=0.01$; Figure 3$)$.

Cortical activation. In concert with our a priori hypotheses, activation peaks in medial prefrontal cortex were found in $\mathrm{AV}-\mathrm{BL}(15,55,36, Z=3.31)$, NA-BL $(-3,55,36$, $Z=3.64 ;-12,39,47, Z=3.34)$ and an activation trend in POS-BL $(-10,39,50, Z=2.59$ trend level) (Figure 4). Multiple activation peaks in response to both aversive and positive emotional content, in comparison to nonaversive content, were found in the visual cortex as expected. Activations in the right and left middle occipital gyri occurred to positive and aversive emotional content, while bilateral cuneus/lingual gyri, bilateral inferior occipital and left fusiform gyri were activated by aversive content only (Figure 2). Occipital cortex activations with aversive stimuli were significantly greater than with positive stimuli.

Direct comparison of positive and aversive conditions. Relative to positive stimuli, aversive stimuli significantly activated larger areas in the visual cortex as expected. Within SLEA, AV-POS comparison produced a small activation peak on the right $(x=-15,-1,-9, Z=2.35)$; however we found no activation in this region in neither AV-NA nor POS-NA comparisons. Thus we have elected to conservatively interpret this focus as a false positive. Relative to aversive stimuli, positive stimuli activated a focus in the left frontal pole. However, we noted a deactivation in this region in the $\mathrm{AV}-\mathrm{NA}$ comparison $(-30,48,7, Z=-4.03)$. Therefore, we performed a VOI analysis of the region for all four conditions (Figure 5). No significant difference between POS and NA conditions was found in this region. Thus, we conclude that the activation found in the POS-AV comparison represents deactivation during the aversive condition.

\section{DISCUSSION}

We hypothesized that activity in SLEA, found in response to salient, aversive stimuli, represented a response to salience in general and not stimulus valence. In accord with this hypothesis, both positive and aversive stimuli activated the SLEA region in the current study. Activation of SLEA to aversive stimuli replicates earlier findings (Liberzon et al, 2000; Taylor et al, 1998), while the activation of SLEA to both aversive and positive stimuli is consistent with the notion of a shared network of regions processing positive and negative emotional stimuli (Breiter and Rosen, 1999; 


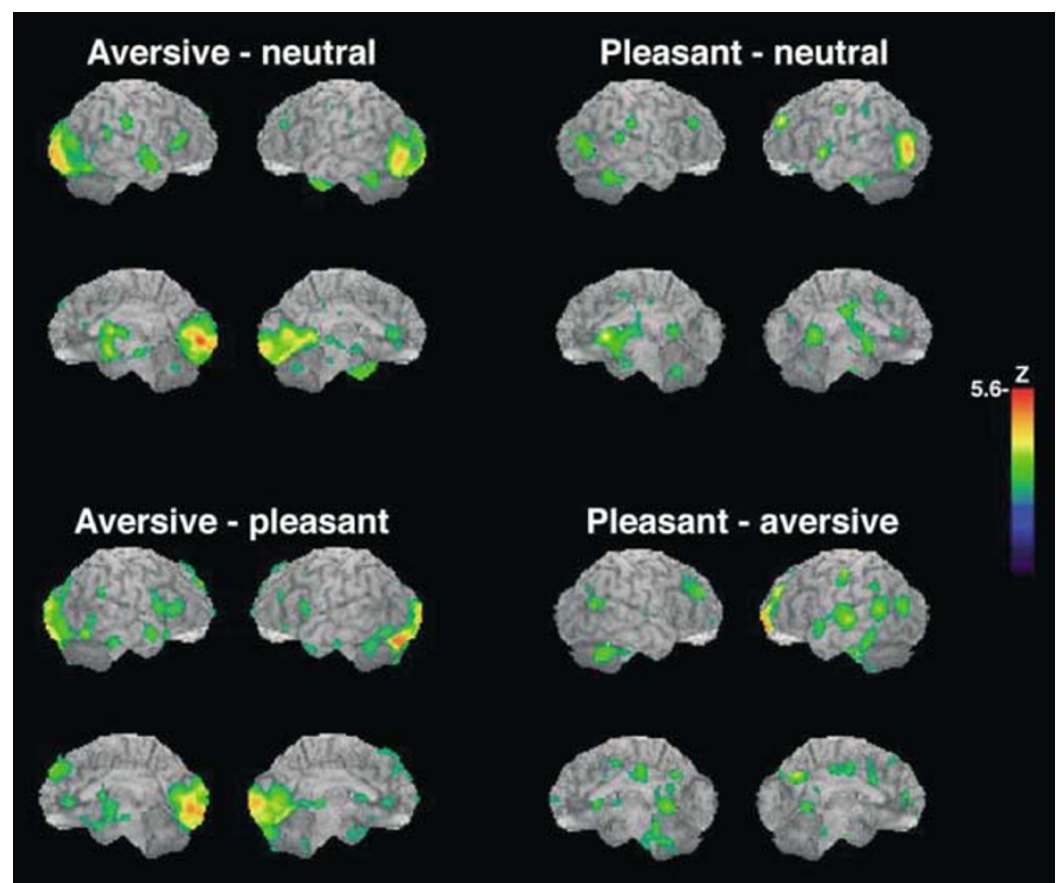

Figure 4 Activity increases in cortical regions while viewing aversive and pleasant stimuli compared to neutral stimuli. The figure shows the activity increase for aversive and positive pictures relative to the neutral pictures in the upper row and aversive vs pleasant pictures (and vice versa) in the lower row, mapped onto surface-rendered maps from a reference MRI in atlas coordinates. For each comparison, the upper two images depict a lateral view and the lower two images depict a medial view. For all comparisons, activation is depicted as Z-scores on the color scale, displayed for Z> I.65.

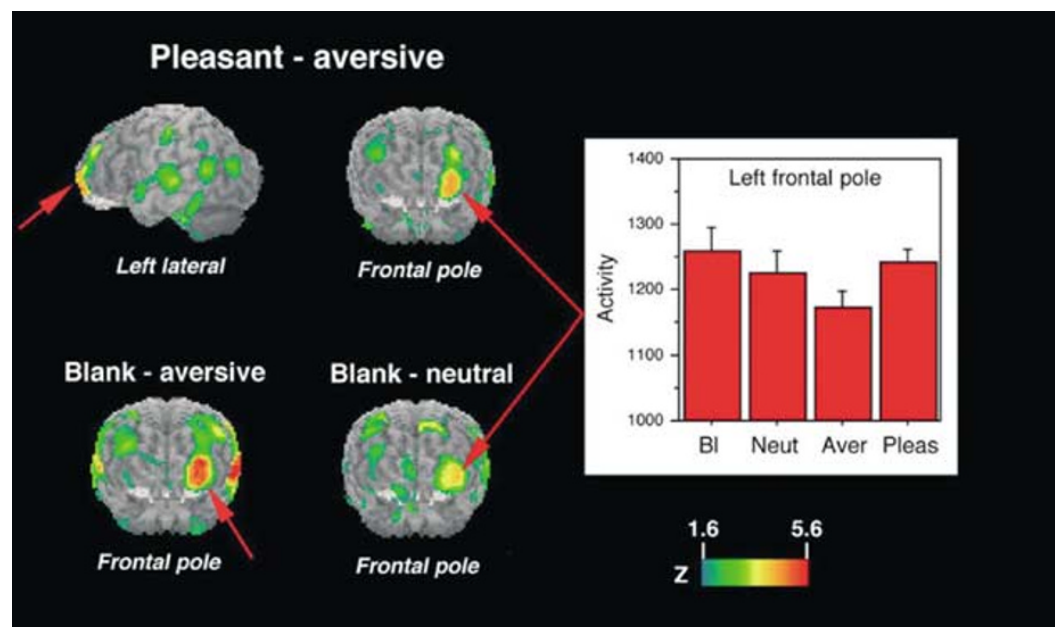

Figure 5 Left frontal pole deactivation. The figure shows a relative activity increase in the left frontal pole for the positive condition, compared to the aversive condition (upper row). The bottom row also shows a relative increase in the same region during the blank condition, compared to the AV and NA, suggesting that observed activations are better characterized as deactivations. The imbedded graph depicts activity (normalized to mean of I00) for all four conditions (AV: aversive; POS: pleasant; NA: nonaversive; Bl: blanks) in the left frontal pole (red arrows). Activity was derived from a spherical volume of interest (I $3.5 \mathrm{~mm}$ diameter), centered on coordinates of the activation peak from the POS-AV subtraction. Surface rendered MRI maps follow the same conventions as Figure I.

Teasdale et al, 1999; Whalen et al, 1998). The shared network that identifies and classifies stimuli as meaningful to an organism's survival can be a useful component of emotional responses, both positive and negative. Determining whether salient stimuli should be approached or avoided defines the particular valence, for example, positive or negative. This process is potentially more economic, allowing early selection of stimuli that require further attention/actions in an environment with a vast number of potentially important cues. This is also entirely consistent with the concept of a shared central process for the assignment of motivational salience, developed by Berridge and Robinson (1998).

Amygdaloid involvement in processing pleasant stimuli is frequently debated in the imaging literature. The role of the amygdala in fear behaviors is well documented (Davis, 1986; LeDoux et al, 1990; Phillips and LeDoux, 1992), and a number of neuroimaging studies reported amygdaloid 
activation with aversive but not pleasant stimuli (Paradiso et al, 1999; Phillips et al, 1997). Some authors have suggested that phylogenetically newer circuits for detection of pleasure would recruit more cortical brain than a primitive, more limbic, danger-detection system (Paradiso et al, 1999). However, ingestive and reproductive functions that provide a substrate for appetitive or pleasure detection are as basic and primary as danger detection, and as likely to be mediated by 'primitive' limbic brain as aversive processing. Indeed, animal studies have demonstrated amygdaloid involvement in appetitive or 'pleasant' functions (Holmes and Egan, 1973; Kondo and Yamanouchi, 1995; Murray et al, 1996; Schoenbaum et al, 1998), and an increasing number of imaging studies have demonstrated amygdaloid activation in response to both pleasant and aversive stimuli (Breiter and Rosen, 1999; Hamann et al, 1999; Whalen et al, 1998; Zalla et al, 2000). Similarly, a broader role in emotional processing has been demonstrated for other structures within the extended amygdala, such as the nucleus accumbens, that participate in defensive or fear-related behaviors (Beck and Fibiger, 1995; Berridge et al, 1999) as well as in reward-related behaviors. Together these data suggest that the amygdaloid region responds to appetitive as well as aversive stimuli, consistent with a hypothesized role in the response to general stimulus salience.

If this conceptualization of the role of extended amygdala region in emotional processing is correct, how does one account for the fact that amygdala is consistently activated by the aversive stimuli in almost all studies, but much less consistently by the pleasant or appetitive stimuli? This is consistent with the concept of negativity bias, whereby aversive stimuli tend to elicit more intense responses than pleasant stimuli (Ito et al, 1998). Indeed, this is one interpretation of the stronger activation foci for AV-NA in SLEA and the visual cortex. It is also possible that aversive, and potentially dangerous, stimuli, are processed as more immediately salient for survival than positive stimuli that usually signal feeding, procreative, or social opportunities. Thus, while salience detection might be common for all stimuli, the potential impact on immediate survival might have shaped the system evolutionarily to identify aversive stimuli as more salient. Since our intention was to demonstrate common activation for pleasant and aversive stimuli, we sought to find the most intense examples of each valence and did not attempt to match our stimulus sets on arousal. While one could interpret our findings as an effect of 'arousal', the concept of arousal is not really an alternative explanation, but can rather be viewed as overlapping with, or subsumed within, the concept of emotional salience.

A number of additional methodological factors potentially contribute to inconsistent amygdaloid responses to pleasant emotions. The majority of in vivo imaging studies are underpowered, thus limiting the ability to interpret negative findings. In addition, cognitive processing, present in almost all activation studies, decreases emotional activation of the amygdaloid region (Drevets, 1998; Liberzon et al, 2000), which would affect responses to all stimuli, and could render a weaker signal to positive stimuli undetectable. The concept of pleasant stimuli is often broadly and liberally used in imaging studies. The range includes stimuli that carry incentive salience (food, smell, sexual arousal) (Berridge, 1996), but also stimuli that are intellectually or aesthetically pleasing (happy faces, beautiful landscapes, etc), and lack direct incentive salience. If SLEA is responsive to incentive salience, stimuli that lack this type of salience would not be expected to activate this region. In the present study, we chose to use IAPS pictures to evoke emotional responses. This specific methodology differs from facial expressions of emotion, such as those used by Whalen et al (1998) in their study, because it directly evokes an emotional response (perception of the stimulus and the subjective experience that follows). Thus, we believe our current findings speak to the role of SLEA in experiencing emotion and not to the proposed roles of this region in detection of ambiguous stimuli or of recognition of emotional expression. On the other hand, it also introduces a confound of complex visual input that is difficult to match in the control condition. We have made a substantial effort to improve this matching (see Methods section), but the potential contribution of visual complexity to the observed activations cannot be entirely discounted. This is particularly true when comparisons to the blank condition are made. However, we note that activations of the dMPFC have been reported for processing emotional material when visual complexity has been matched (Gusnard et al, 2001; Lane et al, 1997a; Taylor et al, 2000; Teasdale et al, 1999).

The anatomical localization of SLEA activation deserves further comment. It is customary in neuroimaging studies to localize activation to the amygdala if an activation focus falls within specific coordinates. The delineation of a functional continuum between amygdaloid nuclei, SLEA, bed nucleus of stria terminalis (BNST), and the shell of nucleus accumbens (Alheid and Heimer, 1988; de Olmos and Heimer, 1999; Heimer et al, 1997b) suggests that activations in the different parts of extended amygdala might subserve a similar functional role. It is interesting to note that the extended amygdala region is comprised of the shell of the nucleus accumbens, implicated in reward mechanisms - rostrally, and of the amygdaloid nuclei, implicated in fear-caudally. It is possible that while the extended amygdala processes general stimulus salience, there is also a rostro-caudal gradient within this region, where more rostral components respond to more appetitive stimuli and more caudal structures to aversive stimuli. A rostro-caudal distinction within the nucleus accumbens for fearful $v s$ appetitive responses has been recently proposed by Reinolds and Berridge (in press) based on direct GABA agonist infusion studies.

In addition to SLEA activation, we also noted other areas of activity for pleasant and aversive stimuli. Activation peaks found in dMPFC replicate previous findings (Gusnard et al, 2001; Lane et al, 1997b; Teasdale et al, 1999), and further support the proposed role for this region in the processing of emotional stimuli. Since all the conditions in the current study included a similar level of cognitive demand (rating the valence), the specific contribution of this region to cognitive modulation of emotional content could not be ascertained. We did find strong modulation of visual cortex by emotional content, replicating previous work using aversive stimuli (Liberzon et al, 2000; Taylor et al, 2000), as well as work using both positive and negative 
stimuli (Lane et al, 1997a; Paradiso et al, 1999). We matched our image sets on luminance, and used black and white images, thus making it unlikely that color composition could affect these results. The direct comparison of pleasant with aversive stimuli revealed an activation focus in the left frontal pole (Figure 3), consistent with the lateralization of valence hypothesis (Davidson, 1995; Natale et al, 1983; Sackeim et al, 1982). However, the region of interest analysis revealed that activities to pleasant, neutral, and blank stimuli were nearly identical, whereas activity decreased in response to aversive stimuli. This provides empirical support to the possibility that right lateralization during positive emotional experience can stem from the deactivation in the left cortical regions as well as from the activation of the right hemisphere.

We did not find activations in orbitofrontal cortex and cerebellum, as reported by others (Bechara et al, 2000; Davidson and Irwin, 1999; Lane et al, 1997a). However, it is possible that our study lacked sufficient power to demonstrate activation of these regions with this particular task. We would not venture interpretations about the possible significance of the apparent absence of activation here. Also, some of the activation peaks reached only trend-level significance, and although post hoc ROI analysis confirmed their significance, these findings need to be considered preliminary, requiring future replication. Finally, it is important to keep in mind that in the sublenticular region of the substantia innominata, cholinergic neurons of the cortico-petal system do not belong to the extended amygdala. Hence, any activation of these neurons in the SLEA peaks reported would not constitute activity of the extended amygdala.

In conclusion, the present study has demonstrated sublenticular/extended amygdala involvement in the processing of both aversive and pleasant emotional components of visual stimuli, consistent with our hypothesis of salience detection and attribution. A better understanding of the specific roles of limbic regions in these processes not only allows us to define neuroanatomical networks involved in emotional responses, but is also essential to our ability to address pathological conditions involving emotional dysregulation, for example, psychiatric disorders. In vivo imaging studies, informed by the most recent basic science research, provide a powerful tool to assist us in this process.

\section{ACKNOWLEDGEMENTS}

This work was supported by the Ann Arbor Veterans Administration Medical Center (IL) and National Alliance for Research in Schizophrenia and Depression (SFT) and the National Institute of Mental Health (SFT-K08 MH01258).

\section{REFERENCES}

Alheid GF, Heimer L (1988). New perspectives in basal forebrain organization of special relevance for neuropsychiatric disorders: the striatopallidal, amygdaloid, and corticopetal components of substantia innominata. Neuroscience 27: 1-39.

Bechara A, Damasio H, Damasio AR (2000). Emotion, decision making and the orbitofrontal cortex. Cereb Cortex 10: 295-307.
Beck CH, Fibiger HC (1995). Conditioned fear-induced changes in behavior and in the expression of the immediate early gene c-fos: with and without diazepam pretreatment. J Neurosci 15: 709-720.

Berridge CW, Mitton E, Clark W, Roth RH (1999). Engagement in a non-escape (displacement) behavior elicits a selective and lateralized suppression of frontal cortical dopaminergic utilization in stress. Synapse 32: 187-197.

Berridge KC (1996). Food reward: brain substrates of wanting and liking. Neurosci Biobehav Rev 20: 1-25.

Berridge KC, Robinson TE (1998). What is the role of dopamine in reward: hedonic impact, reward learning, or incentive salience? Brain Res Brain Res Rev 28: 309-369.

Breiter HC, Etcoff NL, Whalen PJ, Kennedy WA, Rauch SL, Buckner RL et al (1996). Response and habituation of the human amygdala during visual processing of facial expression. Neuron 17: 875-887.

Breiter HC, Rosen BR (1999). Functional magnetic resonance imaging of brain reward circuitry in the human. Ann NY Acad Sci 877: 523-547.

Cherry SR, Woods RP, Mazziotta JC (1993). Improved signal-tonoise in activation studies by exploiting the kinetics of oxygen15-labeled water. J Cereb Blood Flow Metab 13: S714.

Davidson RJ (1995). Cerebral asymmetry, emotion and affective style. In: Davidson RJ, Mugdahl K (eds). Brain Asymmetry. MIT Press: Cambridge, MA. pp 361-387.

Davidson RJ, Irwin W (1999). The functional neuroanatomy of emotion and affective style. Trends Cogn Sci 3: 11-21.

Davis M (1986). Pharmacological and anatomical analysis of fear conditioning using the fear-potentiated startle paradigm. Behav Neurosci 100: 814-824.

Davis M (1992). The role of the amygdala in conditioned fear. In: Aggleton JP (ed). The Amydala: Neurolbiological Aspects of Emotion, Memory and Mental Dysfunction. Wiley-Liss: New York. pp 255-395.

Davis M, Whalen PJ (2001). The amygdala: vigilance and emotion. Mol Psychiatry 6: 13-34.

de Olmos JS, Heimer L (1999). The concepts of the ventral striatopallidal system and extended amygdala. Ann NY Acad Sci 877: $1-32$.

Dolan RJ, Fletcher P, Morris J, Kapur N, Deakin JF, Frith CD (1996). Neural activation during covert processing of positive emotional facial expressions. Neuroimage.

Drevets WC (1998). Functional neuroimaging studies of depression: the anatomy of melancholia. Annu Rev Med 49: 341-361.

Elliott R, Friston KJ, Dolan RJ (2000). Dissociable neural responses in human reward systems. J Neurosci 20: 6159-6165.

Friston KJ, Frith CD, Liddle PF, Frackowiak RS (1991). Comparing functional (PET) images: the assessment of significant change. $J$ Cereb Blood Flow Metab 11: 690-699.

Gallagher M, Schoenbaum G (1999). Functions of the amygdala and related forebrain areas in attention and cognition. Ann NY Acad Sci 877: 397-411.

Ghashghaei HT, Barbas H (2001). Neural interaction between the basal forebrain and functionally distinct prefrontal cortices in the rhesus monkey. Neuroscience 103: 593-614.

Gusnard DA, Akbudak E, Shulman GL, Raichle ME (2001). Medial prefrontal cortex and self-referential mental activity: relation to a default mode of brain function. Proc Natl Acad Sci USA 98: 4259-4264.

Hamann S, Mao H (2002). Positive and negative emotional verbal stimuli elicit activity in the left amygdala. Neuroreport 13: $15-19$.

Hamann SB, Ely TD, Grafton ST, Kilts CD (1999). Amygdala activity related to enhanced memory for pleasant and aversive stimuli. Nat Neurosci 2: 289-293.

Haxby JV, Ungerleider LG, Horwitz B, Maisog JM, Rapoport SI, Grady CL (1996). Face encoding and recognition in the human brain. Proc Natl Acad Sci USA 93: 922-927. 
Heimer L, Alheid GF, de Olmos JS, Groenewegen HJ, Haber SN, Harlan RE et al (1997a). The accumbens: beyond the core-shell dichotomy. J Neuropsychiatry Clin Neurosci 9: 354-381.

Heimer L, Harlan RE, Alheid GF, Garcia MM, de Olmos J (1997b). Substantia innominata: a notion which impedes clinicalanatomical correlations in neuropsychiatric disorders. Neuroscience 76: 957-1006.

Holland PC, Gallagher M (1999). Amygdala circuitry in attentional and representational processes. Trends Cogn Sci 3: 65-73.

Holmes JE, Egan K (1973). Electrical activity of the cat amygdala during sexual behavior. Physiol Behav 10: 863-867.

Ito TA, Larsen JT, Smith NK, Cacioppo JT (1998). Negative information weighs more heavily on the brain: the negativity bias in evaluative categorizations. J Pers Soc Psychol 75: 887-900.

Kalivas PW, Nakamura M (1999). Neural systems for behavioral activation and reward. Curr Opin Neurobiol 9: 223-227.

Kling AS, Brothers L (1992). The amygdala and social behavior. In: Aggleton JP (ed). The Amygdala: Neurobiological Aspects of Emotion, Memory and Mental Dysfunction. Wiley-Liss: New York. pp 353-377.

Kondo Y, Yamanouchi K (1995). The possible involvement of the nonstrial pathway of the amygdala in neural control of sexual behavior in male rats. Brain Res Bull 38: 37-40.

Koob GF (2000). Neurobiology of addiction. Ann NY Acad Sci 909: $170-185$.

Kosslyn SM, Shin LM, Thompson WL, McNally RJ, Rauch SL, Pitman RK et al (1996). Neural effects of visualizing and perceiving aversive stimuli: a PET investigation. Neuroreport 7: 1569-1576.

Lane RD, Fink GR, Chau PM, Dolan RJ (1997a). Neural activation during selective attention to subjective emotional responses. Neuroreport 8: 3969-3972.

Lane RD, Reiman EM, Ahern GL, Schwartz GE, Davidson RJ (1997b). Neuroanatomical correlates of happiness, sadness, and disgust. Am J Psychiatry 154: 926-933.

Lang PJ, Bradley MM, Fitzsimmons JR, Cuthbert BN, Scott JD, Moulder B et al (1998). Emotional arousal and activation of the visual cortex: an fMRI analysis. Psychophysiology 35: 199-210.

LeDoux JE, Cicchetti P, Xagoraris A, Romanski LM (1990). The lateral amygdaloid nucleus: sensory interface of the amygdala in fear conditioning. J Neurosci 10: 1062-1069.

Liberzon I, Taylor SF, Fig LM, Decker LR, Koeppe RA, Minoshima $S$ (2000). Limbic activation and psychophysiologic responses to aversive visual stimuli. Interaction with cognitive task [In Process Citation]. Neuropsychopharmacology 23: 508-516.

Minoshima S, Berger KL, Lee KS, Mintun MA (1992). An automated method for rotational correction and centering of three-dimensional functional brain images. $\mathrm{J} \mathrm{Nucl} \mathrm{Med} \mathrm{33:}$ 1579-1585.

Minoshima S, Koeppe RA, Fessler JA, Mintun MA, Berger KL, Taylor SF et al (1993). Integrated and automated data analysis method for neuronal activation studies using [O-15] water PET Quantification of brain function, tracer kinetics and image analysis in brain PET. Elsevier: Akita. pp 409-415.

Minoshima S, Koeppe RA, Frey KA, Kuhl DE (1994). Anatomic standardization: linear scaling and nonlinear warping of functional brain images. J Nucl Med 35: 1528-1537.

Morris JS, Friston KJ, Dolan RJ (1997). Neural responses to salient visual stimuli. Proc R Soc London Ser B 264: 769-757.
Murray EA, Gaffan EA, Flint Jr RW (1996). Anterior rhinal cortex and amygdala: dissociation of their contributions to memory and food preference in rhesus monkeys. Behav Neurosci 110: $30-42$.

Natale M, Gur RE, Gur RC (1983). Hemispheric asymmetries in processing emotional expressions. Neuropsychologia 21: 555565.

Paradiso S, Johnson DL, Andreasen NC, O'Leary DS, Watkins GL, Ponto LL et al (1999). Cerebral blood flow changes associated with attribution of emotional valence to pleasant, unpleasant, and neutral visual stimuli in a PET study of normal subjects. Am J Psychiatry 156: 1618-1629.

Phillips ML, Young AW, Senior C, Brammer M, Andrew C, Calder AJ et al (1997). A specific neural substrate for perceiving facial expressions of disgust. Nature 389: 495-498.

Phillips RG, LeDoux JE (1992). Differential contribution of amygdala and hippocampus to cued and contextual fear conditioning. Behav Neurosci 106: 274-285.

Rasia-Filho AA, Londero RG, Achaval M (2000). Functional activities of the amygdala: an overview. J Psychiatry Neurosci 25: $14-23$.

Reynolds SM, Berridge CW (2001). Fear and feeding in the nucleus accumbens: shell localization of GABA substrates for defensive behavior and eating behavior. Journal of Neuroscience 21: 32613270 .

Sackeim HA, Greenberg MS, Weiman AL, Gur RC, Hungerbuhler JP, Geschwind N (1982). Hemispheric asymmetry in the expression of positive and negative emotions. Neurologic evidence. Arch Neurol 39: 210-218.

Schoenbaum G, Chiba AA, Gallagher M (1998). Orbitofrontal cortex and basolateral amygdala encode expected outcomes during learning. Nat Neurosci 1: 155-159.

Spitzer RL, Williams JBW, Gibbon M, First MB (1988). Structured Clinical Interview for DSM-III-R. American Psychiatric Press: Washington DC.

Taylor SF, Liberzon I, Fig LM, Decker LR, Minoshima S, Koeppe RA (1998). The effect of emotional content on visual recognition memory: a PET activation study (In Process Citation). Neuroimage 8: 188-197.

Taylor SF, Liberzon I, Koeppe RA (2000). The effect of graded aversive stimuli on limbic and visual activation. Neuropsychologia 38: 1415-1425.

Taylor SF, Phan KL, Decker LR, Liberzon I (in press). Limbic response to emotionally salient stimuli reduced by rating intensity. Neuroimage.

Teasdale JD, Howard RJ, Cox SG, Ha Y, Brammer MJ, Williams SC et al (1999). Functional MRI study of the cognitive generation of affect. Am J Psychiatry 156: 209-215.

Whalen PJ, Rauch SL, Etcoff NL, McInerney SC, Lee MB, Jenike MA (1998). Masked presentations of emotional facial expressions modulate amygdala activity without explicit knowledge. $J$ Neurosci 18: 411-418.

Worsley KJ, Evans AC, Marrett S, Neelin P (1992). A threedimensional statistical analysis for CBF activation studies in human brain. J Cereb Blood Flow Metab 12: 900-918.

Zalla T, Koechlin E, Pietrini P, Basso G, Aquino P, Sirigu A et al (2000). Differential amygdala responses to winning and losing: a functional magnetic resonance imaging study in humans. Eur J Neurosci 12: 1764-1770. 